\title{
Conflict resolution using enhanced label combination method for complex activity recognition in smart home environment
}

\begin{abstract}
Activity recognition in smart home environment is becoming challenging when it is involving more than one resident living in the same space. It is not merely recognizing and tracking the multi-resident activity, but the interaction between them are also need to address in order to provide the great autonomous ambient intelligence $(\mathrm{AmL})$ system. It is a challenging task due to diversity and complexity level of human activity and resident interaction using only binary data from ambient-based type sensors. Strong approach is needed to identify types of interaction based on activity performed either it is individual, parallel or cooperative. Previously, researchers tend to simplify the problem and define the parallel as individual activity due to the sensors type are unobtrusive and open to noise in nature. Hence, we address this issue as separate interaction. This research presents the rule-based approach to recognize complex activity recognition in multi-resident scenario in a smart home setting. It has been tested on the real smart home datasets using multi-label classification technique using Enhanced Label Combination method with random forest as its base classifier. The quality of the classification is selected as evaluation metrics to measure the proposed solution.
\end{abstract}

Keyword: Conflict resolution; Multi-label classification; Smart home environment; Multiresident; Complex activity recognition 